\title{
Study of fission using multi-nucleon transfer reactions
}

\author{
Katsuhisa Nishio ${ }^{1, \star}$, Kentaro Hirose ${ }^{1}$, Mark Vermeulen ${ }^{1}$, Hiroyuki Makii ${ }^{1}$, Riccardo Orlandi ${ }^{1}$, \\ Kazuaki Tsukada ${ }^{1}$, Masato Asai ${ }^{1}$, Atsushi Toyoshima ${ }^{1}$, Tetsuya K. Sato ${ }^{1}$, Yuichiro Nagame ${ }^{1}$, \\ Satoshi Chiba ${ }^{2}$, Yoshihiro Aritomo ${ }^{3}$, Shouya Tanaka ${ }^{3}$, Tsutomu Ohtsuki ${ }^{4}$, Igor Tsekhanovich ${ }^{5}$, \\ Costel M. Petrache ${ }^{6}$, and Andrei Andreyev ${ }^{7,1}$ \\ ${ }^{1}$ Advanced Science Research Center, Japan Atomic Energy Agency, 2-4 Shirakata, Tokai, Naka-gun, Ibaraki \\ 319-1195, Japan \\ ${ }^{2}$ Laboratory for Advanced Nuclear Energy, Tokyo Institute of Technology, 2-12-1, Ookayama, Meguro-ku, \\ Tokyo 152-8550, Japan \\ ${ }^{3}$ Faculty of Science and Engineering, Kindai University, Higashi-Osaka 577-8502, Japan \\ ${ }^{4}$ Research Reactor Institute, Kyoto University, Kumatori-cho, Sennangun, Osaka 590-0494, Japan \\ ${ }^{5}$ University of Bordeaux, 351 Cours de la Libération, 33405 Talence Cedex, France \\ ${ }^{6}$ Centre des Sciences Nucléaire et des Sciences de la Matière, Université Paris-Saclay, CNRS/IN2P3, 91406 \\ Orsay, France \\ ${ }^{7}$ Department of Physics, University of York, Heslington, York, YO10 5DD, UK
}

\begin{abstract}
Multi-nucleon transfer channels of the reactions of ${ }^{18} \mathrm{O}+{ }^{232} \mathrm{Th},{ }^{18} \mathrm{O}+{ }^{238} \mathrm{U}$, ${ }^{18} \mathrm{O}+{ }^{248} \mathrm{Cm}$ were used to measure fission-fragment mass distribution for various nuclides and their excitation energy dependence. Predominantly asymmetric fission is observed at low excitation energies for all the studied cases, with an increase of the symmetric fission towards high excitation energies. Experimental data are compared with predictions of the fluctuation-dissipation model, where effects of multi-chance fission (neutron evaporation prior to fission) was introduced. It was shown that a reliable understanding of the observed fission fragment mass distributions can be obtained only invoking multi-chance fissions.
\end{abstract}

\section{Introduction}

Nuclear fission is usually described as an evolution of a nuclear shape on a potential-energy surface which results from the subtle interplay of macroscopic nuclear properties and microscopic shell effects. Also dynamical effects should have an important role to explain various aspects in fission. New experimental techniques and associated new data are indispensable to further understand fission mechanism. Fission-fragment mass distribution (FFMD) is one of the most fundamental data, which exhibits asymmetric shape at low excitation energy due to shell structures. Traditionally, neutron- and charged particle capture reactions as well as spontaneous fission have been used to study low-energy fissions.

Around 2000, GSI in Darmstadt developed a Coulex-induced fission of relativistic RIBs in inverse kinematics, where comprehensive fission studies were performed for several tens of nuclei in the

${ }^{\star}$ e-mail: nishio.katsuhisa@jaea.go.jp 
neutron-deficient Ac-U region [1]. The recent SOFIA experiment at GSI also followed the same approach but with a much improved technique [2]. Recently, $\beta /$ EC delayed fission was investigated for the very proton-rich nucleus using radioactive beams, and ${ }^{180} \mathrm{Hg}$ was found to show an asymmetric fission as a new region of mass-asymmetric fission [3]. Advancement of new technique for fission studies is reviewed in [4].

Multi-nucleon transfer (MNT) reactions is another unique reaction which allow us to populate neutron-rich nuclei which cannot be accessed by other reactions such as particle capture and/or heavyion fusion reactions. Furthermore, excited states of $\mathrm{CN}$ range widely from under the fission barrier to higher energies, allowing us to measure the excitation energy dependence of FFMDs. Recently, an inverse kinematics technique was applied in the MNT channels of the ${ }^{238} \mathrm{U}+{ }^{12} \mathrm{C}$ reaction to study fission using the large-acceptance magnetic spectrometer VAMOS at GANIL [5, 6]. In these experiments, sufficiently-high $A$ and $Z$ resolution for FFs was achieved due to their kinematic boost, allowing the simultaneous measurement of the complete mass- and atomic-number distributions of fission fragments (FFs).

At the tandem accelerator facility of the Japan Atomic Energy Agency (JAEA), we studied the MNT channels of the reactions ${ }^{18} \mathrm{O}+{ }^{232} \mathrm{Th},{ }^{238} \mathrm{U},{ }^{248} \mathrm{Cm}$ in normal kinematics to obtain FFMDs and their excitation-energy dependence for various isotopes (data for ${ }^{18} \mathrm{O}+{ }^{232} \mathrm{Th}$ were published in [7]). An obvious advantage of this method is a relatively easy possibility to change the projectile and/or the target nuclei. In particular by using targets of the rarest highly-radioactive neutron-rich isotopes heavier than ${ }^{238} \mathrm{U}$ (e.g. $\mathrm{Cm}$ and $\mathrm{Cf}$ ), nuclei to be studied can be extended to isotopes far heavier than uranium, which cannot be used at the accelerator facilities for the inverse kinematics experiments similar to VAMOS or SOFIA.

\section{Experimental methods}

An ${ }^{18} \mathrm{O}$ beam was supplied by the JAEA-tandem accelerator at a typical beam intensity of about $0.5 \mathrm{pnA}$. Beam energies were $157-162 \mathrm{MeV}$, depending on the different run. Targets were prepared by electrodeposition of oxide-target material on a thin Ni backing. Thickness of the target-material layer was around $35-148 \mu \mathrm{g} / \mathrm{cm}^{2}$.

For the event-by-event identification of the transfer channel (thus, of the fissioning nucleus) and of respective coincident FFs, a detection system consisting of a $\Delta \mathrm{E}-\mathrm{E}$ silicon detector telescope and four multiwire proportional chambers (MWPC) were used, see Fig. 1. Specific transfer channels were identified by detecting projectile-like (ejectile) nuclei in twelve $75 \mu$ m-thick trapezoidal $\Delta \mathrm{E}$ silicon detectors which were mounted in a cone around the beam axis, each with the azimuthal angle acceptance of $\Delta \phi=22.5^{\circ}$. After passing through the $\Delta \mathrm{E}$ detector, the ejectiles impinged on the $300 \mu \mathrm{m}$-thick annular silicon strip detector (E-detector), divided in 16 annular strips, which allows determination of the scattering angle $\theta$. The inner and outer radius of the detector are $24.0 \mathrm{~mm}$ and $48.0 \mathrm{~mm}$, respectively, corresponding to the acceptance angle $\theta$ between $16.7^{\circ}$ and $31.0^{\circ}$ relative to the beam direction.

Figure 2 shows the $\Delta E-E_{\text {tot }}$ spectrum for ejectiles obtained in the ${ }^{18} \mathrm{O}+{ }^{232} \mathrm{Th}$ reaction, where the parabolic lines correspond to different transfer channels, including a clear separation of specific isotopes. Isotopic assignment was done in respect of the elastically-scattered peak of ${ }^{18} \mathrm{O}$ and the missing line of ${ }^{8} \mathrm{Be}$. It was further confirmed with the energy-loss calculation. The data from $\Delta E$ $E_{\text {tot }}$ spectra were also used to deduce the excitation energy of the respective fissioning nuclei, which were determined from reaction Q-value and the measured (angle-dependent) ejectile energy $E_{\text {tot }}$. In this procedure we assumed that no excitation energy is given to the ejectile, thus the quoted excitation energies should be considered as upper limits only.

The coincident FFs resulting from the fission of excited nuclei after the MNT reaction are detected by four $200 \times 200 \mathrm{~mm}^{2}$ position-sensitive MWPCs (see in Fig. 1). The MWPCs were operated with 

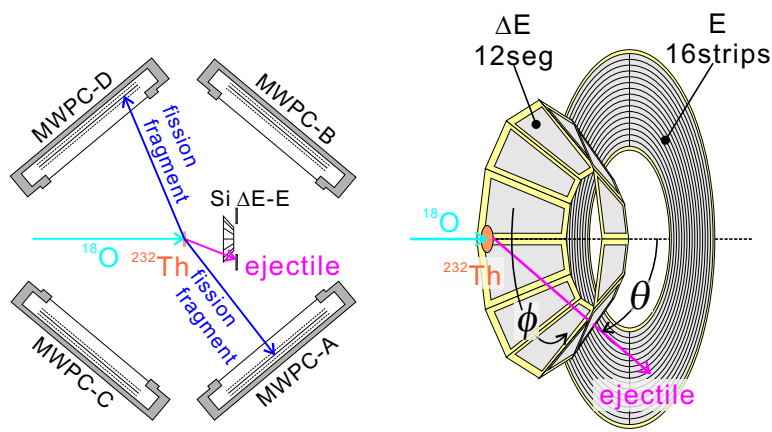

Figure 1. Schematic detection set-up (left) and expanded view of the silicon $\Delta \mathrm{E}-\mathrm{E}$ detector telescope (right). See text for details.

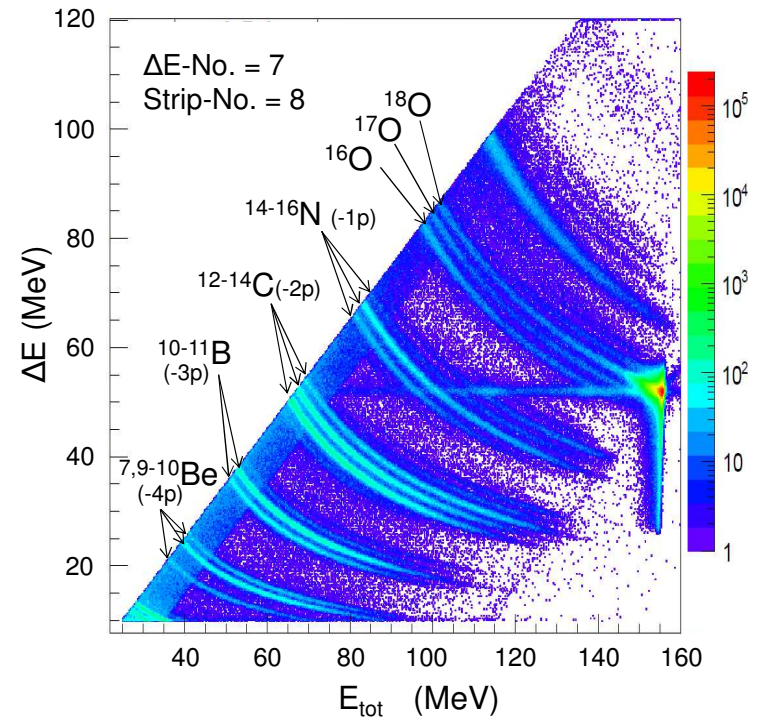

Figure 2. $\Delta E-E_{\text {tot }}$ spectrum for ejectiles measured by one pair of the $\Delta \mathrm{E}$-E detectors obtained in the ${ }^{18} \mathrm{O}+{ }^{232} \mathrm{Th}$ reaction [7]. The curves corresponding to different ejectiles are marked with the respective isotopes. The scattered ${ }^{18} \mathrm{O}$ is also seen in the plot.

an isobutane gas of about 1.5 Torr [8]. The distance between the target and the center of the cathode was $224 \mathrm{~mm}$, and each MWPC covers a solid angle of $0.67 \mathrm{sr}$. The positions of FFs's incidence on the MWPC were determined with a position resolution of $4.0 \mathrm{~mm}$. Charge induced in the cathode of the MWPC was recorded to separate FFs from other reaction products. Typical rise time of the MWPC is $5 \mathrm{~ns}$. Both FFs were detected in coincidence with a pair of MWPC facing both sides of the 


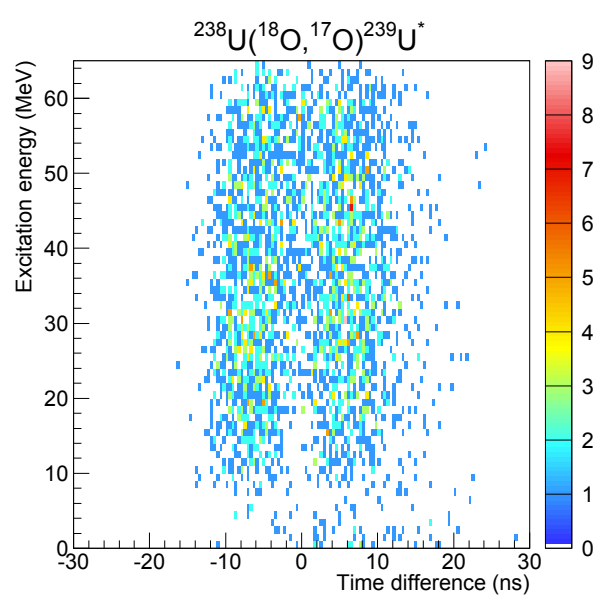

Figure 3. Fission events recorded on the time difference between the signals from coincided MWPCs and excitation energy obtained in one neutron transfer reaction ${ }^{238} \mathrm{U}\left({ }^{18} \mathrm{O},{ }^{17} \mathrm{O}\right){ }^{239} \mathrm{U}^{*}$.

target, $\left(+50.1^{\circ},-129.9^{\circ}\right)$ or $\left(-50.1^{\circ},+129.9^{\circ}\right)$ relative to the beam direction. Fission-fragments time difference, $\Delta T$, between two coincident MWPCs were measured to determine the masses of both FFs. Figure 3 shows an example of recorded FFs on the time difference and excitation energy in the transfer channel of ${ }^{238} \mathrm{U}\left({ }^{18} \mathrm{O},{ }^{17} \mathrm{O}\right){ }^{239} \mathrm{U}^{*}$. Two regions are clearly observed in the low-excitation fissions, corresponding to the light- and heavy-fragment groups, which smear at high-excitation energies.

\section{Results}

FFs masses were determined event-by-event from the kinematic analysis, where the measured $\Delta T$ values and incident positions of both FFs were used. The momentum of the target-like fissioning recoil nucleus is determined by the measured momentum of ejectile under the assumption of a binary reaction process. Figure 4 shows the comparison of FFMDs for ${ }^{239} \mathrm{U}^{*}$, populated in the ${ }^{238} \mathrm{U}\left({ }^{18} \mathrm{O},{ }^{17} \mathrm{O}\right){ }^{239} \mathrm{U}^{*}$ reaction [9], with $\mathrm{n}+{ }^{238} \mathrm{U}[10]$. The obtained FFMDs from MNT reactions agree well with the neutron-induced data, particularly the mass asymmetry at the peak positions at the lowest energy data and the increase of the symmetric fission with excitation energy are noteworthy. The result demonstrates that ${ }^{18} \mathrm{O}$-induced neutron-transfer reaction can be a surrogate of neutron-induced fission to give FFMDs. In Fig. 4, FFMDs of ${ }^{237-238} \mathrm{U}^{*}$, populated both by the ${ }^{238} \mathrm{U}\left({ }^{18} \mathrm{O},{ }^{19-18} \mathrm{O}\right){ }^{237-238} \mathrm{U}^{*}$ reactions and by the ${ }^{232} \mathrm{Th}\left({ }^{18} \mathrm{O},{ }^{13-12} \mathrm{C}\right){ }^{237-238} \mathrm{U}^{*}$ reactions are shown. Fairly good agreement are found for each nuclide and its excitation energy dependence, indicating the insensitivity of the FFMDs to the number of transferred nucleons. It is also noted that the FFMD data for ${ }^{233} \mathrm{~Pa}^{*},{ }^{233} \mathrm{Th}^{*}$ and ${ }^{236} \mathrm{U}^{*}$ from the MNT reactions of ${ }^{18} \mathrm{O}+{ }^{232} \mathrm{Th}$ [7] agree with literature data obtained in proton- and neutroninduced fissions [11-14].

Figure 5 shows the FFMDs for nuclei of ${ }^{238-240} \mathrm{U}^{*},{ }^{239-241} \mathrm{~Np}^{*},{ }^{241-243} \mathrm{Pu}^{*}$; selection from the MNT-channels of the ${ }^{18} \mathrm{O}+{ }^{238} \mathrm{U}$ reaction [9]. The FFMDs of the ${ }^{240} \mathrm{U}^{*},{ }^{240,241} \mathrm{~Np}^{*}$ were obtained for the first time in this experiment. For the other nuclei, the known FFMD data were systematically extended to excitation energies as high as $60 \mathrm{MeV}$. It follows from Fig. 5 that mass-asymmetric fission dominates at low excitation energies for all the measured nuclei. The yield in the mass-symmetric 


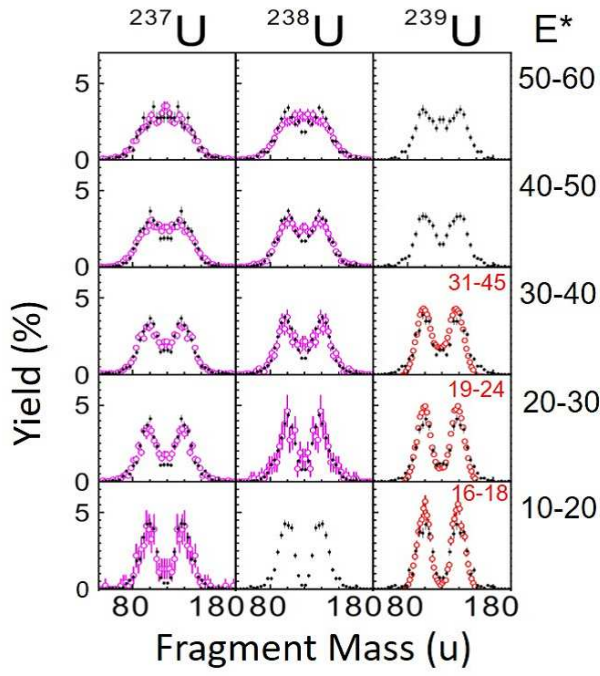

Figure 4. Experimental FFMDs (black dots with error bars) obtained in the ${ }^{238} \mathrm{U}\left({ }^{18} \mathrm{O},{ }^{19-17} \mathrm{O}\right){ }^{237-239} \mathrm{U}^{*}$ reactions. Excitation energy ranges are indicated on the right side. Data are compared with those from the $n+{ }^{238} U[10]$ (open red circles) from the similar excitation energies (shown by the red character). Data for fissions of ${ }^{237,238} \mathrm{U}^{*}$ are compared with the MNT fissions of ${ }^{232} \mathrm{Th}\left({ }^{18} \mathrm{O},{ }^{13-12} \mathrm{C}\right){ }^{237-238} \mathrm{U}^{*}$ [7] (open magenta circles).

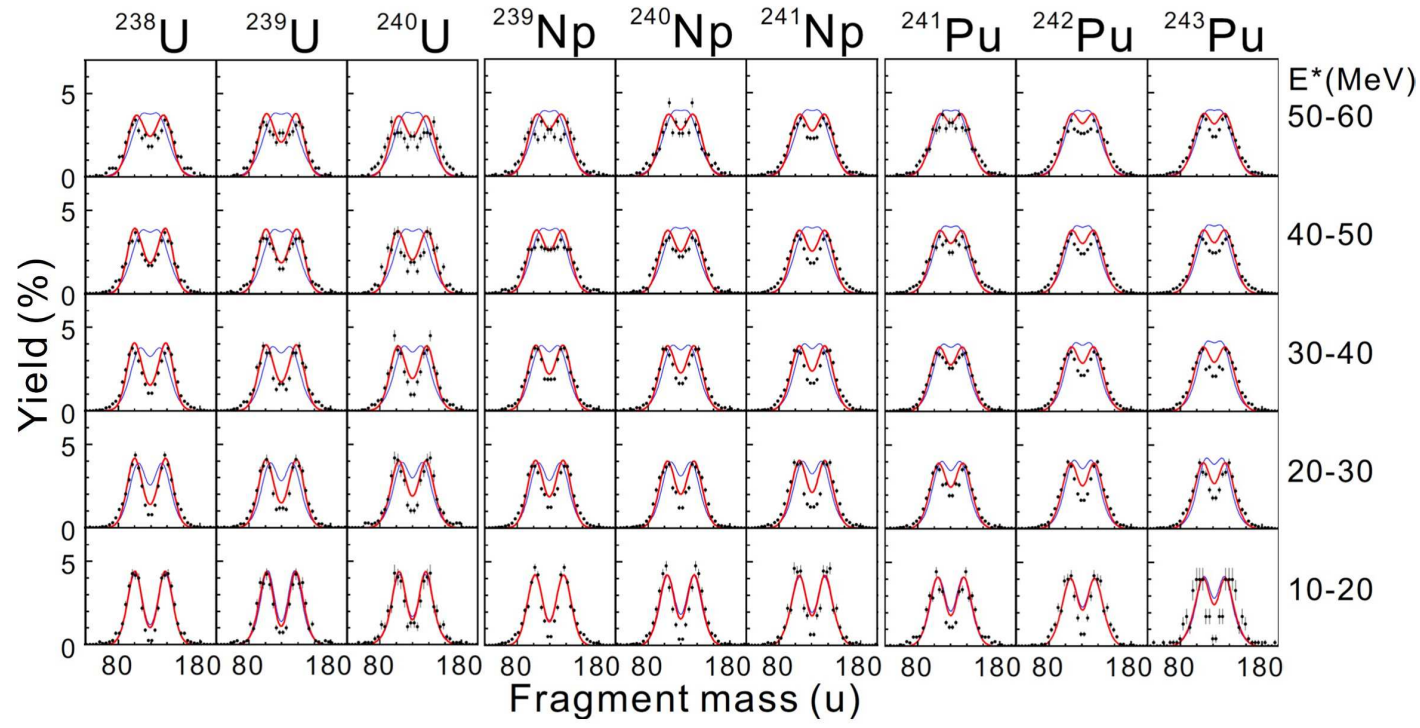

Figure 5. Experimental FFMDs (points with error bars) of the U, Np and Pu isotopes and their dependence on the excitation energy in the range of $E^{*}=10-60 \mathrm{MeV}$. The experimental FFMDs are compared with the Langevin calculations without (blue curves) and with (red curves) the inclusion of the multi-chance fission (see text). 
fission region increases with excitation energy (see also Fig. 4) and the double-peaked shapes tend to be washed out. However, even at the highest energies, $E^{*}=50-60 \mathrm{MeV}$, double-peak structure is preserved for all the studied nuclides in Fig. 5. It is also interesting to note that the measured spectra reveal smaller peak-to-valley ratio in the FFMDs for heavier elements as can be seen, for example, in the spectra of $E^{*}=30-40 \mathrm{MeV}$. All these features will be discussed in the following subsection in comparison with a fluctuation-dissipation fission model.

In the recent measurement of ${ }^{18} \mathrm{O}+{ }^{248} \mathrm{Cm}$, new FFMDs of eleven nuclei are further generated, ${ }^{247,249} \mathrm{Cm},{ }^{249,250,251,252} \mathrm{Bk},{ }^{251,253} \mathrm{Cf},{ }^{254,256} \mathrm{Es}$, and ${ }^{255} \mathrm{Fm}[15]$.

The evolution of the center of the light- and heavy-fragment groups $\left(\bar{A}_{\mathrm{L}}\right.$ and $\left.\bar{A}_{\mathrm{H}}\right)$ with the mass of the $\mathrm{CN}$ in low energy fissions of $10<E^{*}<20 \mathrm{MeV}$ is shown in Fig. 6, where data obtained from the three MNT reactions, ${ }^{18} \mathrm{O}+{ }^{232} \mathrm{Th},{ }^{18} \mathrm{O}+{ }^{238} \mathrm{U}$, and ${ }^{18} \mathrm{O}+{ }^{248} \mathrm{Cm}$ are used. It is found that the $\bar{A}_{\mathrm{H}}$ values are kept constant around 141 , whereas $\bar{A}_{\mathrm{L}}$ increases linearly with mass of fissioning nucleus. The trend shows the dominant influence of the shell structure in heavy fragments, well-known in fission studies.

\section{Discussions}

The measured FFMDs from the MNT reactions are compared with calculations based on the fluctuation-dissipation model developed in [16], where description of fission in Langevin equations from the low-excited state were attempted, and a good reproduction of the measured FFMDs for ${ }^{234,236} \mathrm{U}^{*}$ and ${ }^{240} \mathrm{Pu}^{*}$ from $E^{*}=20 \mathrm{MeV}$ was obtained. As described in [16], the nuclear shape and the corresponding energy is calculated by a two-center shell model [17]. The nuclear shape is defined by three parameters (distance between two potential centers, deformation of fragments, and mass-asymmetry), and the corresponding energy is given by a sum of the liquid-drop energy $V_{\mathrm{LD}}$ and the shell correction energy $V_{\text {shell }}$. The latter term is represented as $V_{\text {shell }}(0) \exp \left(-E^{*} / E_{\mathrm{d}}\right)$ using the shell correction energy at the zero temperature $V_{\text {shell }}(0)$ and shell damping parameter $E_{\mathrm{d}}$, where $E_{\mathrm{d}}=20 \mathrm{MeV}$ was chosen as in [16]. For simplicity, we assumed that the total excitation energy of the system after the MNT reactions is given to the initial excitation energy $\left(E^{*}\right)$ of the fissioning nucleus.

The results of Langevin calculation are shown in Fig. 5 by thin blue curves. Calculated FFMDs are broadened with the experimental mass resolution $(\sigma=\sim 6.5 \mathrm{u})$. Under this assumption, the mass asymmetry, i.e. the peak positions of the double-humped FFMD, for all isotopes are reproduced below $E^{*}=20 \mathrm{MeV}$ with clear deviations seen for higher energies. At the highest energy, the calculation shows structure-less symmetric fission in contrast to the measurement. It is seen that the peak-tovalley ratio is reproduced only for the uranium isotopes, ${ }^{238-240} \mathrm{U}$ of $E^{*}=10-20 \mathrm{MeV}$, as well as nuclei ${ }^{231-234} \mathrm{Th},{ }^{232-236} \mathrm{~Pa}$ and ${ }^{234-237} \mathrm{U}$ [7] studied in the ${ }^{18} \mathrm{O}+{ }^{232} \mathrm{Th}$ reaction. For the neptunium and plutonium isotopes of $E^{*}=10-20 \mathrm{MeV}$, the calculated peak-to-valley ratio is smaller than the experiments. One of the possible reasons for the deviation could be in the treatment of the neck parameter $\epsilon(0<\epsilon<1)$ [17] to define the shape of nucleus, where $\epsilon=0.35$ was adopted in the present calculation.

In the above calculation we assumed that all the fission events originate from the initial excitation energy populated by MNT channel. As a next step we attempted to take into account the multi-chance fission (MCF). It is defined as a fission occurring after neutron emission from $\mathrm{CN}$, thus FFs from low-excited and neutron-less excited residual nucleus can contribute (second chance fission). When the residual excited nucleus has enough high excitation energy, further competition between neutronevaporation and fission (third chance fission) can take place. The higher chance fission successively occurs until the competition terminates. The experimentally observed FFMD is represented by a superposition of all the possible fission chances. These features are demonstrated by Fig. 7, which compares the experimental data for fission of ${ }^{240} \mathrm{U}^{*}$ at the initial excitation energy $E^{*}=40-50 \mathrm{MeV}$ 


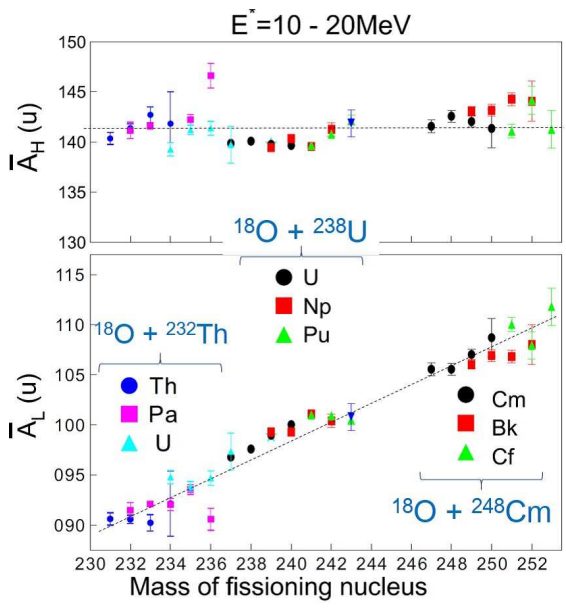

Figure 6. Center of the light and heavy fragment groups $\left(\bar{A}_{\mathrm{L}}\right.$ and $\left.\bar{A}_{\mathrm{H}}\right)$ as a function of mass of the fissioning nuclei in low-excitation fission of $10<E^{*}<20 \mathrm{MeV}$. Data are obtained from the reactions of ${ }^{18} \mathrm{O}+{ }^{232} \mathrm{Th},{ }^{238} \mathrm{U}$ and ${ }^{248} \mathrm{Cm}$ using the same setup shown in Fig. 1.

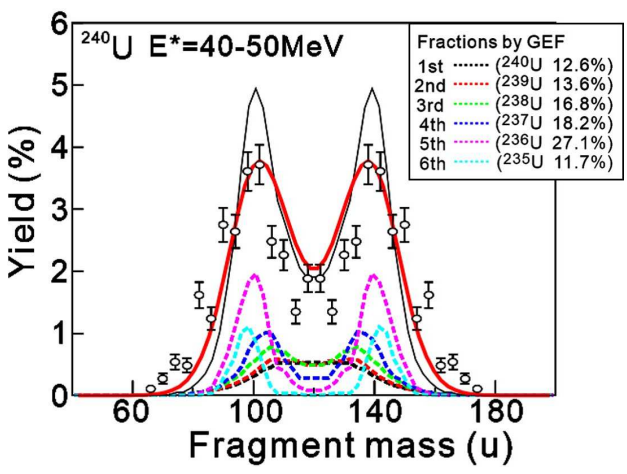

Figure 7. Experimental FFMD of ${ }^{240} \mathrm{U}^{*}$ (open circles) measured at the initial excitation energy of $40-50 \mathrm{MeV}$ obtained from the two neutron-transfer channel ${ }^{238} \mathrm{U}\left({ }^{18} \mathrm{O},{ }^{16} \mathrm{O}\right){ }^{240} \mathrm{U}^{*}$, is compared with the Langevin calculation [16] taking into account multi-chance fission. Thin gray curve is obtained by summing all the fission-chances, which is then broadened with the experimental mass resolution (red curve).

with the Langevin calculation taking into account the MCF. As the excitation energy for the calculation, the middle value $45 \mathrm{MeV}$ for the bin-width (40-50 MeV) was used. Probabilities for each fission chances were calculated by the GEF code (Version 2015v2.2) [18], where spins of the compound nucleus were set at zero for simplicity. The reduction of the excitation energy of the compound nucleus due to the neutron emission was determined from neutron binding energies [19] and a mean energy of the emitted neutron, $\sim 1.9 \mathrm{MeV}$, obtained by the PACE2 code [20]. At each step of MCF, the potential energy surface for the respective compound nucleus was adopted. The finally calculated FFMD shown by the thin gray line is the sum of the FFMDs over the possible chance fissions. It reproduces the observed peak positions of the experimental FFDM, but has narrower peaks than the measured 
ones. However, after introducing the experimental mass resolution $(\sigma=6.5 \mathrm{u})$ as shown by the thick solid curve, the calculation well reproduces also the peak-to-valley ratio and the total width of the FFMD. It is seen that at this initial energy, the 1st- and 2nd-chance fission occur with somewhat lower probabilities, which exhibits more symmetric-like fission. On the contrary, the higher-fission chances, after emission of several neutrons (2-5, in this case), lead predominantly to an asymmetric mass split. It is evident that the mass-asymmetric fission observed in the data even at the high excitation energy originates from the lower-energy 4th-, 5th-, and 6th-chance fissions $\left({ }^{235,236,237} \mathrm{U}\right)$ [9].

The same calculation procedure was applied for all the cases as displayed in Fig. 5, and the results are shown by the red thick curves. In contrast to the calculation without MCF (thin blue curves), the calculation with MCF well explains the variation of FFMDs with the excitation energies. Also massasymmetry and peak-to-valley ratio observed at the higher excitation energies are well reproduced. The calculation also demonstrates the decreasing peak-to-valley ratio of FFMDs for heavier elements (from uranium to plutonium), observed for example in the $E^{*}=30-40 \mathrm{MeV}$ range, whereas the analysis without MCF predicts almost the same distributions through the isotopes. It should also be noted that the consideration of MCF validates that the shell effect responsible for mass-asymmetric fission disappears around $E^{*}=30-40 \mathrm{MeV}$ (blue curves in Fig. 5), resulting from the shell-damping energy $E_{\mathrm{d}}=20 \mathrm{MeV}$ entering in the excitation-energy dependence of the shell correction energy $V_{\text {shell. }}$.

\section{Summary and outlook}

It is shown that the multi-nucleon transfer reaction is a powerful tool to study fission for nuclei which cannot be accessed by particle-capture and/or heavy-ion fusion reactions. An advantage in the normal kinematics is that the nuclei to be studied can be significantly expanded by using available highpurity radioactive targets. Fission studies using the MNT reactions with other targets, such as ${ }^{243} \mathrm{Am}$, ${ }^{231} \mathrm{~Pa}$, and ${ }^{249} \mathrm{Cf}$, are planned at the JAEA tandem facility. Furthermore, a reaction using the ${ }^{254} \mathrm{Es}$ target will allow us to study low-energy fissions of fermium isotopes, where sharp transition from the mass-asymmetric fission (e.g. ${ }^{256} \mathrm{Fm}$ ) to the sharp symmetric fission (e.g. ${ }^{258} \mathrm{Fm}$ ) was observed in the spontaneous fission studies [21].

In addition to investigate the fission-fragment properties, a measurement of prompt neutrons in coincidence with FFs has stated to obtain neutron multiplicity $\bar{v}(A)$ from individual fragments with mass $A$ and their excitation energy dependence, by mounting a neutron detector array around the present fission setup.

Special thanks are due to the crew of the JAEA tandem facility for their beam operation. Present study is supported by "Comprehensive study of delayed-neutron yields for accurate evaluation of kinetics of high-burn up reactors" and "Development of prompt-neutron measurement in fission by surrogate reaction method and evaluation of neutron-energy spectra" by the Ministry of Education, Culture, Sports, Science and Technology of Japan (MEXT).

\section{References}

[1] K.-H. Schmidt et al., Nucl. Phys. A 665, 221 (2000)

[2] E. Pellereau et al., Phys. Rev. C 95, 054603 (2017)

[3] A.N. Andreyev et al., Phys. Rev. Lett. 105, 252502 (2010)

[4] A.N. Andreyev et al., Rep. on Prog. Phys., in print

[5] M. Caamaño et al., Phys. Rev. C 92, 034606 (2015) 
[6] C. Rodríguez-Tajes et al., Phys. Rev. C 89, 024614 (2014)

[7] R. Léguillon et al., Phys. Lett. B, 761, 125 (2016) Ibid., M. Wang et al., 1603

[8] K. Nishio et al., Phys. Rev. C 77, 064607 (2008)

[9] K. Hirose et al., submitted

[10] V.D. Simutkin et al., Nucl. Data Tables 119, 331 (2014)

[11] S.I. Mulgin et al., Nucl. Phys. A 824, 1 (2009)

[12] I. Nishinaka, et al., Phys. Rev. C, 70, 014609 (2004), and Proceedings of the Fourth International Conference on Fission and Properties of Neutron-rich Nuclei, Sanibel Island, Florida, USA, 11-17 Nov. 2007, pp. 206-211, World Scientific

[13] I.V. Ryzhov et al., Phys. Rev. C 83, 054603 (2011)

[14] Ch. Straede, B. Jørgensen and H.-H. Knitter, Nucl. Phys. A 462, 85 (1987)

[15] K. Nishio et al., International Conference on Nuclear Data for Science and Technology (ND2016), 11-16 Sep. 2016, Bruges, Belgium

[16] Y. Aritomo and S. Chiba, Phys. Rev. C 88, 044614 (2013)

[17] K. Sato et al., Z. Phys. A 288, 383 (1978)

[18] K.-H. Schmidt and B. Jurado and C. Amouroux and C. Schmitt, Nuclear Data Sheets 131, 107 (2016)

[19] P. Möller et al., Atomic Data and Nuclear Data Tables. 109-110, 1 (2016)

[20] A. Gavron, Phys. Rev. C 21, 230 (1980)

[21] D.C. Hoffman, Nucl. Phys. A 502, 21c (1989) 
